\title{
Contribución al estudio de las reacciones de hidratación del cemento portland por espectroscopía infrarroja
}

\section{Estudio de la hidratación acelerada del cemento portland}

T. VAZQUEZ Y A. RUIZ DE GAUNA

IETCC

\section{INTRODUCCION}

En trabajos anteriores (1), (2), (3) y (4) se estudiaron por espectroscopía infrarroja las fases sintetizadas del clínker, el propio clínker, el cemento hidratado hasta una edad de 4 años y con distintas proporciones de yeso y $\mathrm{CaCl}_{2}$, hasta una edad de 28 días.

En el presente trabajo, con el fin de poner de manifiesto, principalmente, la evolución de la tobermorita y de los sulfoaluminatos cálcicos, se ha realizado el proceso de la "hidratación acelerada" de un cemento portland.

\section{PREPARACION DE MUESTRAS}

La hidratación acelerada (5) se realizó como sigue:

En un recipiente cónico de $20 \mathrm{~cm}$ de diámetro y de altura $8 \mathrm{~cm}$, con cierre hermético, se mezclan $250,0 \mathrm{~g}$ de cemento y $500 \mathrm{ml}$ de agua. En el recipiente se ponen también $250 \mathrm{~g}$ de bolas de acero inoxidable para una perfecta agitación. Inmediatamente después de la mezcla del agua con el cemento, se cierra el envase y se coloca en una máquina vibradora, diseñada especialmente para ello; se mantiene en vibración continua durante 72 horas y se filtra la "lechada" resultante. El sólido que retiene el filtro se seca a vacío. Posteriormente a los cementos hidratados y pulverizados en mortero, se les introdujo en un recipiente con agua; para evitar que las muestras entraran en contacto directo con ésta, se las colocó sobre una placa de porcelana porosa que servía de separación entre sólido y líquido; a continuación se hizo un vacío de unos $5 \mathrm{~mm}$ de $\mathrm{Hg}$, hasta conseguir en el ambiente $100 \%$ de h. r. Inmediatamente se incorporó al recipiente $\mathrm{CO}_{2}$ hasta una presión ligeramente superior a la atmosférica, cosa que se consiguió con un globo de goma conectado en la llave del recipiente. Se mantiene la muestra en esas condiciones durante 72 horas. 
Las muestras resultantes de la hidratación (tanto normal como acelerada) del cemento se estudiaron por espectroscopía IR, con el apoyo de Análisis Térmico, según el orden siguiente:

1. Hidratado normalmente (figura 1; espectro n." 1).

2. Hidratado aceleradamente, en ambiente de $\mathrm{N}_{2}$ (figura 1 ; espectro $n$." 2 ).

3. Hidratado aceleradamente, en ambiente de $\mathrm{N}_{2}$ y mantenido posteriormente en ambiente saturado de $\mathrm{CO}_{2}$ durante 72 horas (figura 1; espectro $n .{ }^{\circ} 3$ ).

1'. Hidratado normalmente (figura 2 ; espectro n." 1).

2. Hidratado aceleradamente, en ambiente de $\mathrm{CO}_{2}$ (figura 2 ; espectro n." 2 ).

3'. Hidratado aceleradamente, en ambiente de $\mathrm{CO}_{2}$ y mantenido posteriormente en ambiente saturado de $\mathrm{CO}_{2}$ durante 72 horas (figura 2; espectro n." 3 ).

Todas las muestras han sido tratadas además con el método de metanol-salicílico ("Takashima") (6); los espectros correspondientes se dan en las figuras 3 y 4.

El análisis del cemento empleado se da en la Tabla I.

T AB L A I

Análisis químico de la muestra de cemento empleada

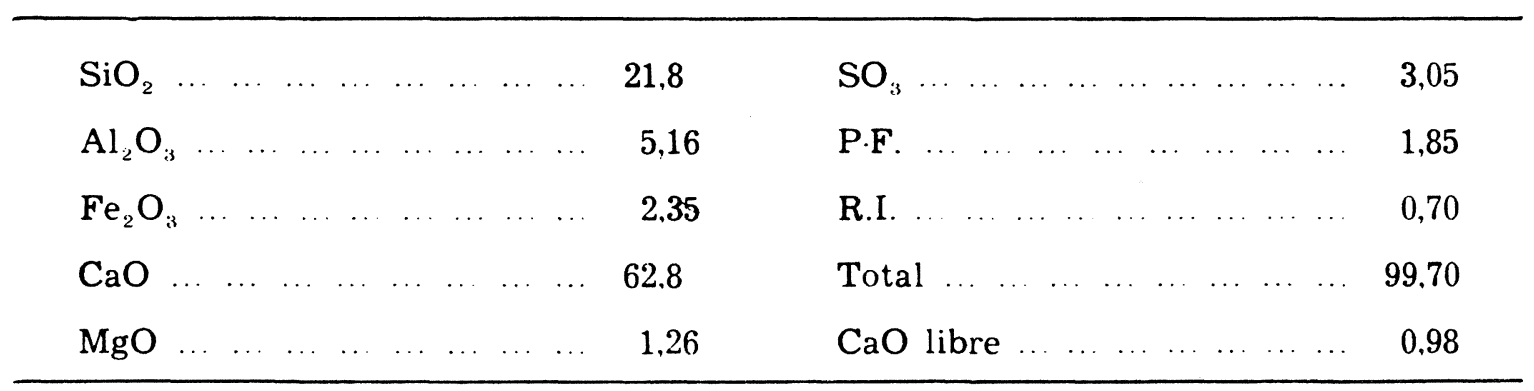

(Valores expresados en \% en peso y referidos a la muestra seca a $105^{\prime \prime}-110^{\circ} \mathrm{C}$ ).

\section{RESULTADOS}

En el espectro IR del cemento portland hidratado normalmente (figuras 1 y 2 ; espectro n." 1) se comprueba la hidratación de los silicatos por formarse tobermorita (banda a $970 \mathrm{~cm}^{-1}$ ) y como es lógico $\mathrm{Ca}(\mathrm{OH})_{2}$ (banda muy aguda a $3.640 \mathrm{~cm}^{-1}$ ). La banda indicativa de la tobermorita es ancha y poco intensa, lo que hace suponer que la tobermorita formada es aún rica en $\mathrm{Ca}^{2+}$. Es conocido el hecho de que la tobermorita tiene aniones silicatos en distintos grados de condensación y que los geles tobermoríticos contienen aniones $\mathrm{SiOH}^{-}$, que al reaccionar entre sí aumentan el número de cadenas $\mathrm{Si}-\mathrm{O}-\mathrm{Si}$. Hasta cierto límite, la unión de aniones entre sí depende de la relación $\mathrm{CaO} / \mathrm{SiO}_{2}$ en la tobermorita. En el caso aquí estudiado se deduce que los iones $\mathrm{Ca}^{2+}$ están en gran parte aún unidos a los aniones silícicos, de tal forma que la hidrólisis fue poco avanzada, habiéndose formado, por consiguiente. poco $\mathrm{Ca}(\mathrm{OH})_{2}$. como se comprueba por la pequeña intensidad de su banda a $3.640 \mathrm{~cm}$ '.

Aparecen los sulfatos hidratados (ettringita), lo que se demuestra por las bandas de absorción de $1.100 \mathrm{~cm}^{-1}$ y las de vibración de valencia y deformación de agua. 

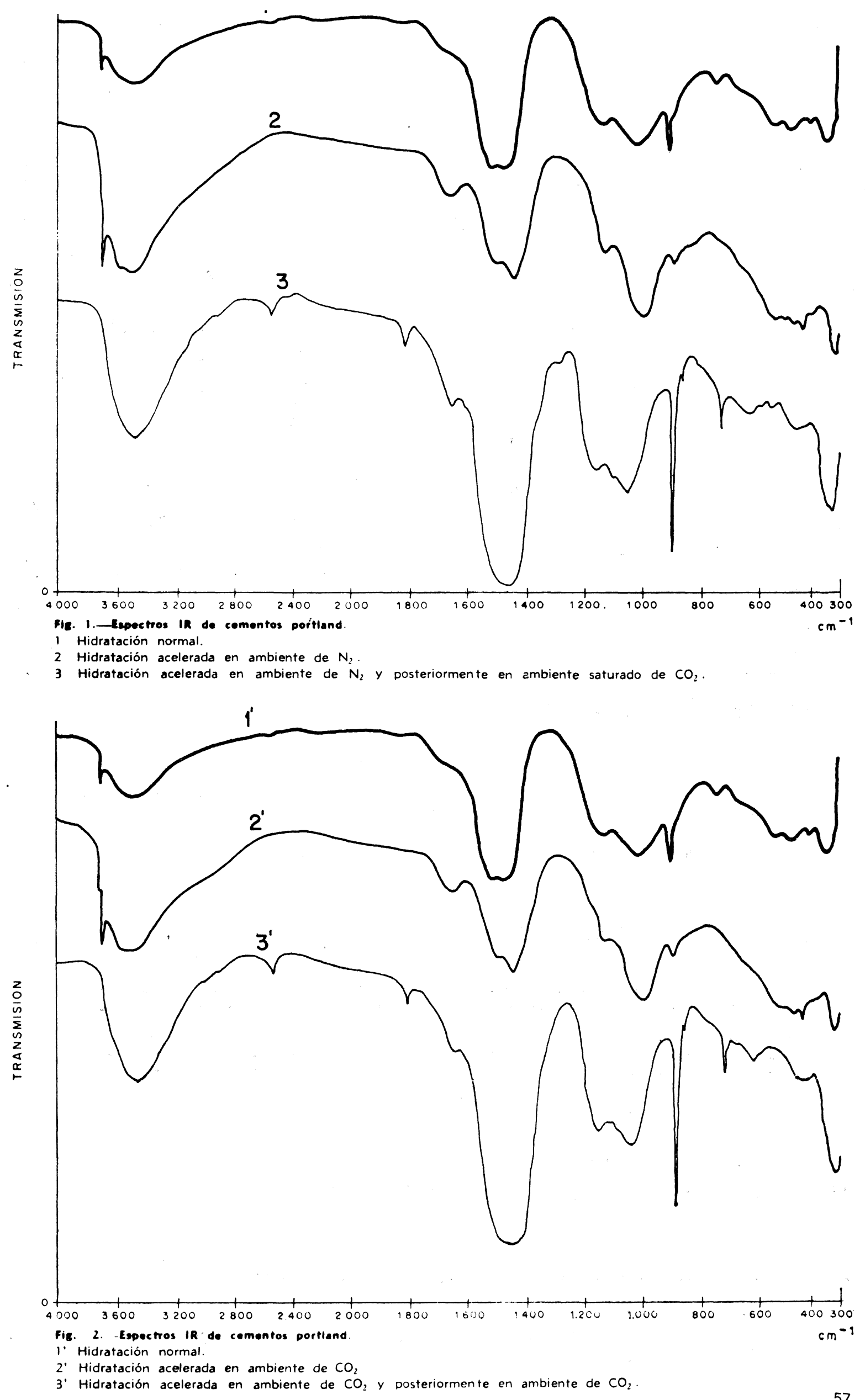


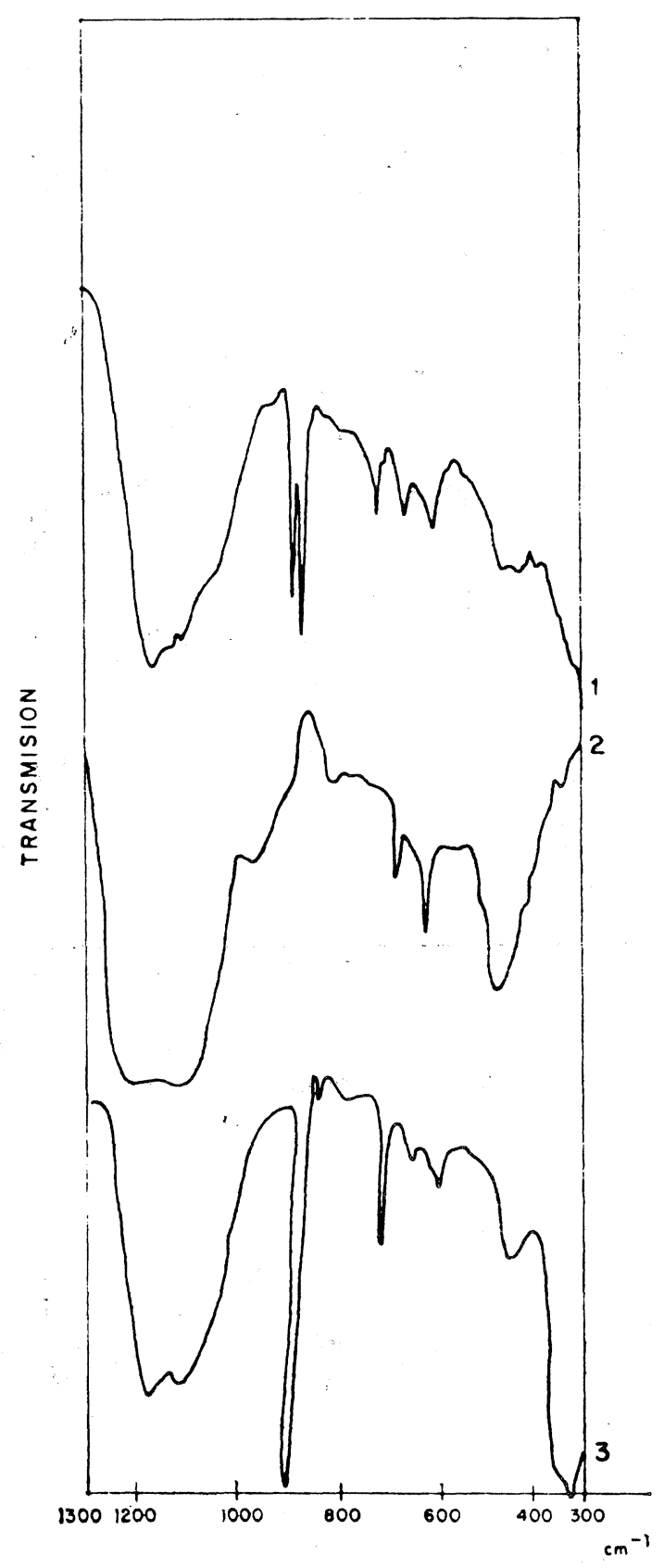

Fir. 3-Eepectros IR de cementos hidratados posteriormente tratados con el métode "Takashiman.

1 Hidratación normal.

2 Hidratación acelerada en ambiente de $\mathrm{N}_{2}$.

3 Hidratación acelerada en ambiente de $\mathrm{N}_{2}$ y posteriormente en ambiente de $\mathrm{CO}_{2}$.

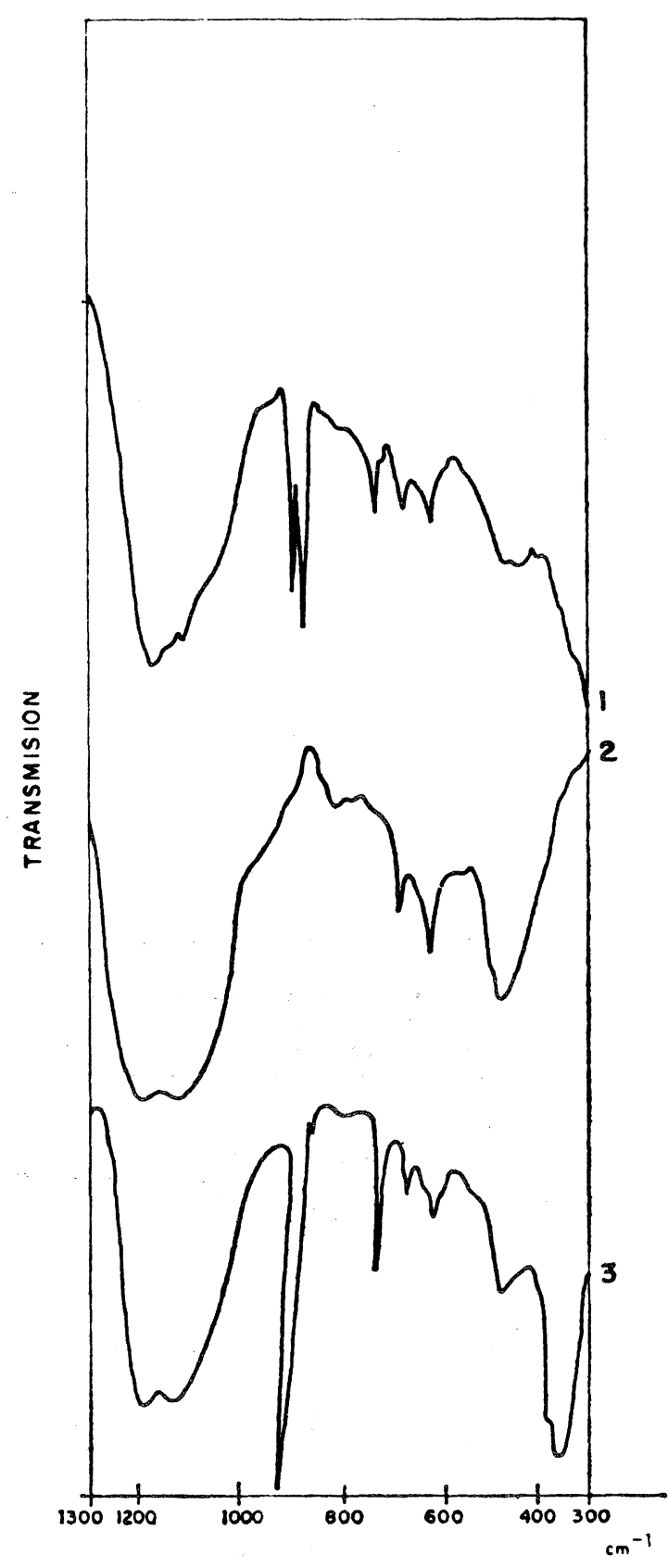

Fis. 4.- Espectros It de comentes ididatados y pesteriormente tratados con el método Takashimon.

1 Hidratación normal.

2 Hidratación acelerada en ambiente de $\mathrm{CO}_{2}$.

3 Hidratación acelerada en ambiente de $\mathrm{CO}_{2}$ y posteriormente en ambiente de $\mathrm{CO}_{2}$.

Por otra parte, aparece $\mathrm{CaCO}_{3}$ cristalizado en forma de vaterita.

\subsection{Cemento portland hidratado aceleradamente en ambiente de $\mathbf{N}_{2}$}

En el espectro $n^{\circ} 2$ (figura 1) se aprecia un cambio notable que viene dado por el proceso de hidratación acelerada. 
Aun cuando se procuró evitar la contaminación del $\mathrm{CO}_{2}$ mediante un flujo constante de $\mathrm{N}_{2}$ durante el tiempo de amasado y envasado de la pasta de cemento, el espectro IR de la muestra estudiada aparece con bandas de absorción características de $\mathrm{CO}_{3}{ }^{2-}$. Incluso como se comprueba en las curvas 2 de las figuras 1 y 2 , las muestras hidratadas en atmósfera de $\mathrm{N}_{2}$ y de $\mathrm{CO}_{2}$ son semejantes. Sin embargo los espectros de dichas muestras sometidas a un tratamiento posterior con $\mathrm{CO}_{2}$ presentan diferencias.

En la muestra correspondiente al espectro n." 2. figura 1, aparecen bandas de carboaluminatos, (hombro a $3.698 \mathrm{~cm}^{-1}$, bandas débiles anchas entre 3.400 y $3.600 \mathrm{~cm}^{-1}$ bandas a $1.418 \mathrm{~cm}^{-1}$, banda a $\left.420 \mathrm{~cm}^{-1}\right)$. Hay $\mathrm{Ca}(\mathrm{OH})_{2}$ en cantidad considerablemente superior a la que se vió en el espectro del cemento normalmente hidratado. Los carbonatos cálcicos aparecen en un estado de cristalización pobre.

La banda característica de la tobermorita es más intensa y su estado de cristalización aparente, más perfecto. Esta interpretación se confirma por la disminución de la intensidad de la banda a $525 \mathrm{~cm}^{-1}$. Ello, unido a la icantidad notablemente superior, de $\mathrm{C}_{\varepsilon}(\mathrm{OH})_{2}$, demuestra que la polimerización tetraédrica ha sido mayor, así como el ordenamiento estructural. No obstante, la cristalización no ha sido suficiente para que, por difracción de rayos $\mathrm{X}$, se detecte en forma clara la estructura cristalina de la tobermorita.

Los sulfoaluminatos se aprecian alterados al haber evolucionado hacia otras formas con distinto grado de hidratación, como se ha puesto de manifiesto en los espectros de las muestras tratadas por el método de Takashima, lo que se detalla al final del presente apartado. Por A. T. se demuestra la existencia de monosulfoaluminato cálcico hidratado (fig. 5).

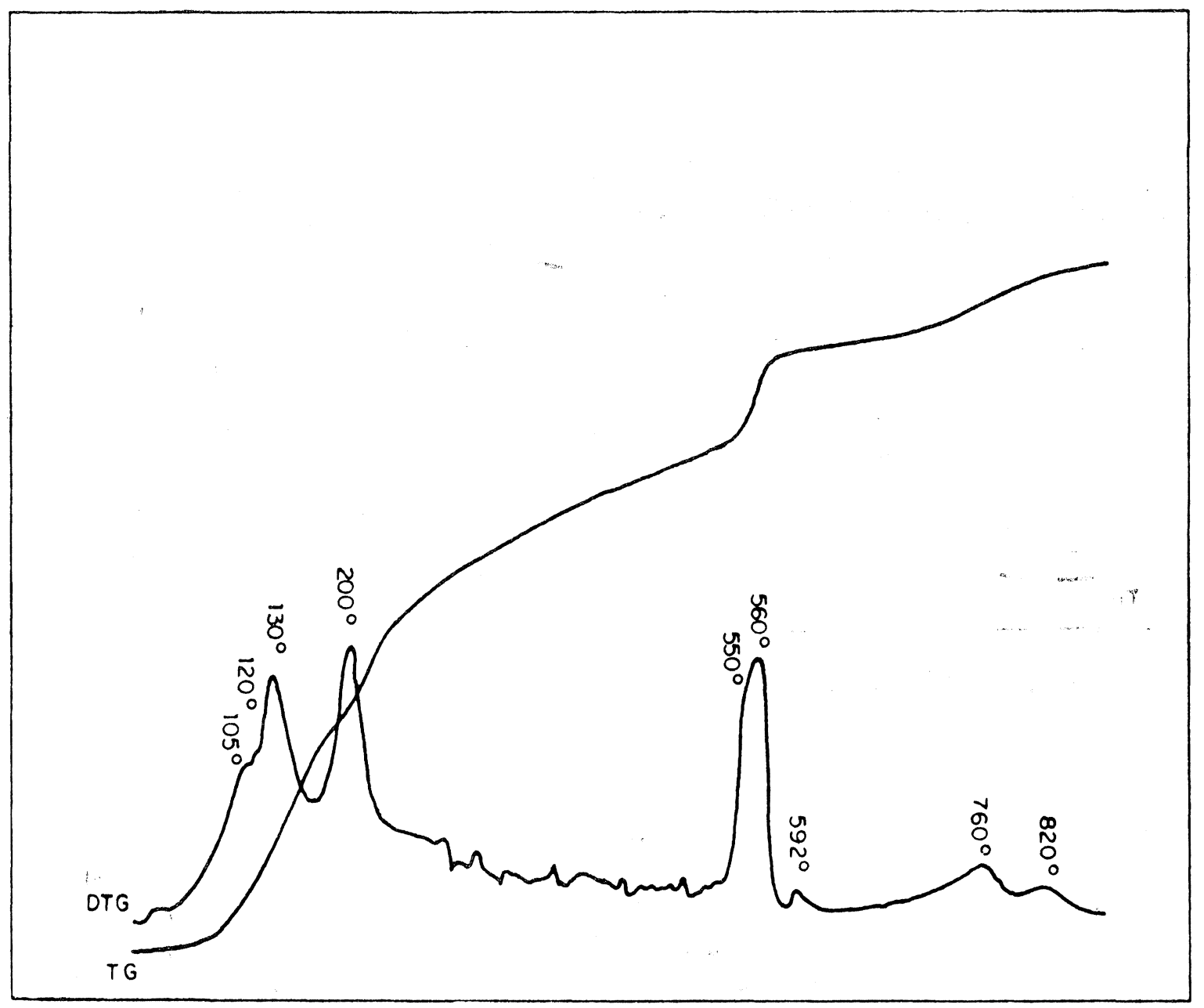

Fig. S.-A.T. del cemento hidratado acoloradamente. Ambiente de $N_{2}$ 
En el espectro n. 3 se ven cambios que demuestran la gran influencia que ejerce el $\mathrm{CO}_{2}$ sobre el cemento hidratado.

Como era de esperar, no se aprecian las bandas características del $\mathrm{Ca}(\mathrm{OH})_{2}$ por encontrarse este compuesto totalmente combinado con el $\mathrm{CO}_{2}$ y los silicatos. Las bandas de vibración de valencia y deformación del agua aparecen sencillas, siendo muy intensa la primera, a $3.430 \mathrm{~cm}^{-1}$, y muy distinta a la observada en los casos anteriores la segunda, a $1.640 \mathrm{~cm}^{-1}$. Por otra parte en A.T. se observa que el pico en $130^{\circ}$ de la muestra n. 2 se desplazó en la n..$^{\circ} 3$ (fig. 6) hasta $149^{\circ}$ y aparece en esta muestra un nuevo pico en $725^{\circ}$. Este hecho encaja con la evolución de la banda IR en $1.640 \mathrm{~cm}^{-1}$ que aparecía en la muestra 2 y que era debida al agua de constitución de la "mezcla" de $\mathrm{C}_{4} \mathrm{AH}_{13}$, del monosulfoaluminato $\mathrm{y}$ de tobermorita, que en la muestra 3 no aparecen.

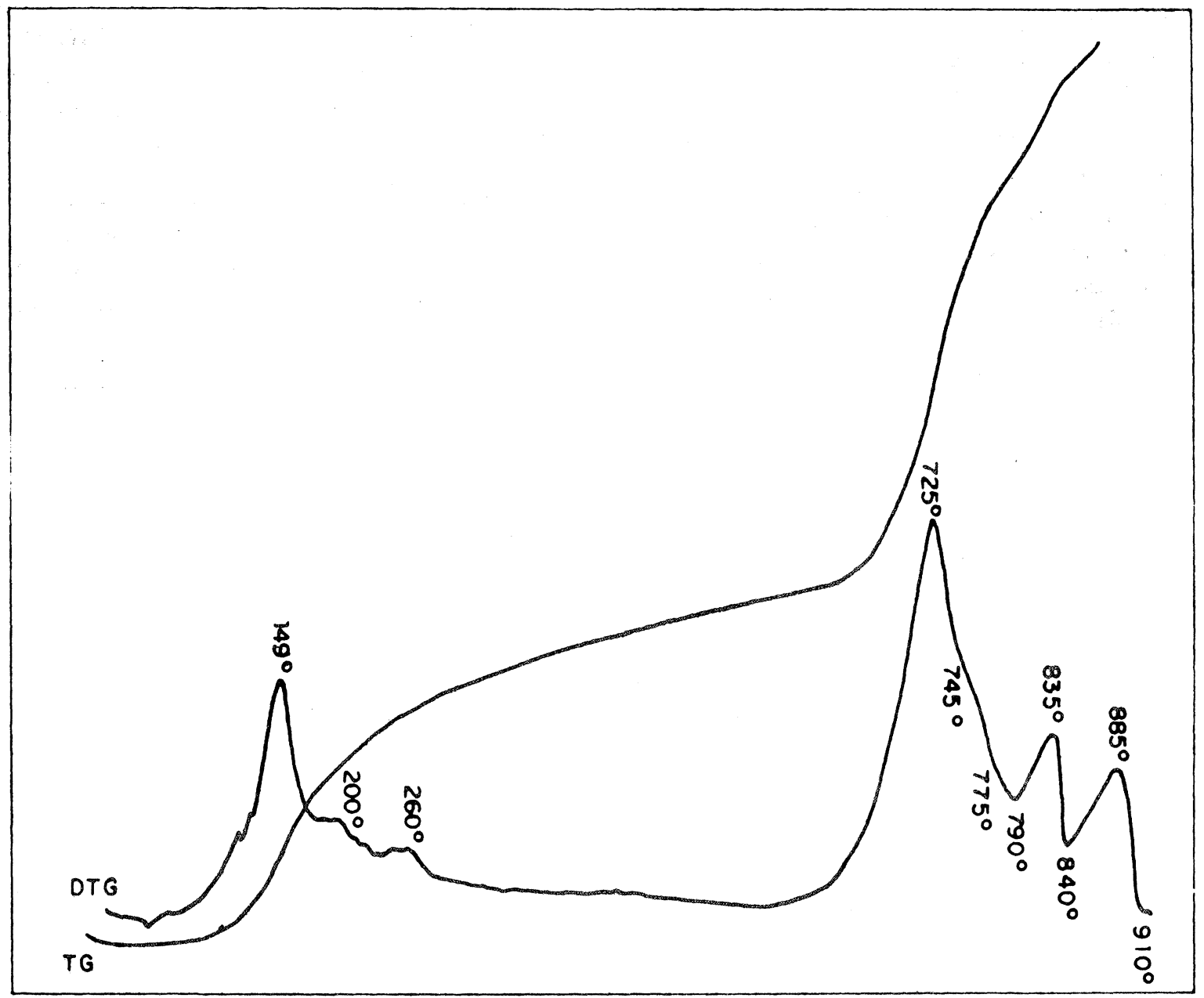

Fig. 6.-A.T. del cemento hidratado aceleradamente. Ambiente de $N_{2} \checkmark$ posteriormente mantenido en ambiente saturado de $\mathrm{CO}_{2}$.

Aun cuando los sulfoaluminatos se estudiarán en la muestra tratada con metanol-salicílico, se ve en el espectro IR n. 3 que la banda que en el $\mathrm{n}^{\circ} 2$ aparecía hacia $1.100 \mathrm{~cm}^{-1}$, se desplazó a número de onda más alto $\left(1.132 \mathrm{~cm}^{-1}\right)$. Todo lo anterior lleva a deducir que ha habido formación de $\mathrm{CaSO}_{4} \cdot 1 / 2 \mathrm{H}_{2} \mathrm{O}$, lo que se explica como consecuencia de una parcial destrucción del monosulfoaluminato cálcico causada por la acción del $\mathrm{CO}_{2}$ sobre la muestra. Este resultado no se opone al obtenido por A.T. (fig. 6). 
La banda más característica de la fase tobermorítica aparece desplazada a mayor frecuencia (hasta $1.025 \mathrm{~cm}^{-1}$ ). Las características de la fase ferrítica desaparecen también. Se observan. sin embargo, bandas en 540 y $570 \mathrm{~cm}^{-1}$ de $\mathrm{AlO}_{6}$ y en $1.280 \mathrm{~cm}^{-1}$ que atribuimos a Si-O-Al. En análisis térmico hay salida simultánea de $\mathrm{CO}_{2}$ y de $\mathrm{OH}$. a una temperatura inferior, por supuesto, que la del $\mathrm{CO}_{2}$ procedente de carbonatos cálcicos o silicocarbonatos anhidros. La interpretación que hacemos de estos hechos es que el $\mathrm{CO}_{2}$, en parte, se incluye en la red tobermorítica, con el resultado de hacer una policondensación de silicato y formación de $\mathrm{CaCO}_{3}$. Como hipótesis de trabajo esquematizamos este hecho de la siguiente manera:

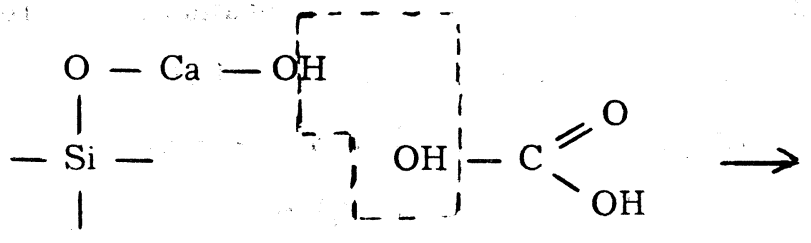

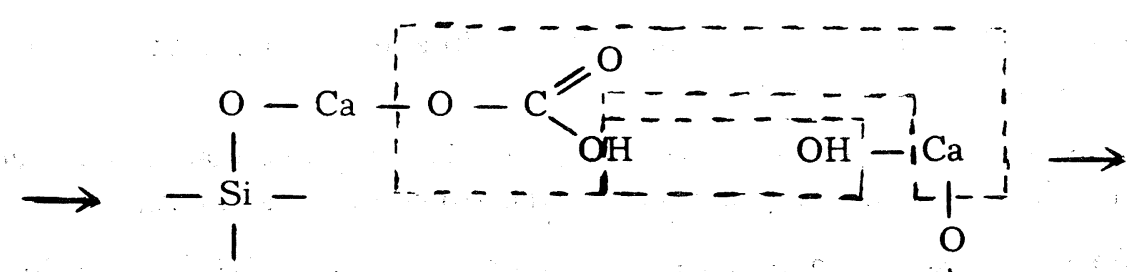

$$
\begin{aligned}
& -\mathrm{Si}- \\
& \longrightarrow-\frac{1}{1}-\mathrm{O}-\mathrm{Ca}-\mathrm{O}-\frac{1}{1}-\mathrm{Si}-+\mathrm{CaCO}_{3}+\mathrm{H}_{2} \mathrm{O}
\end{aligned}
$$

o bien:

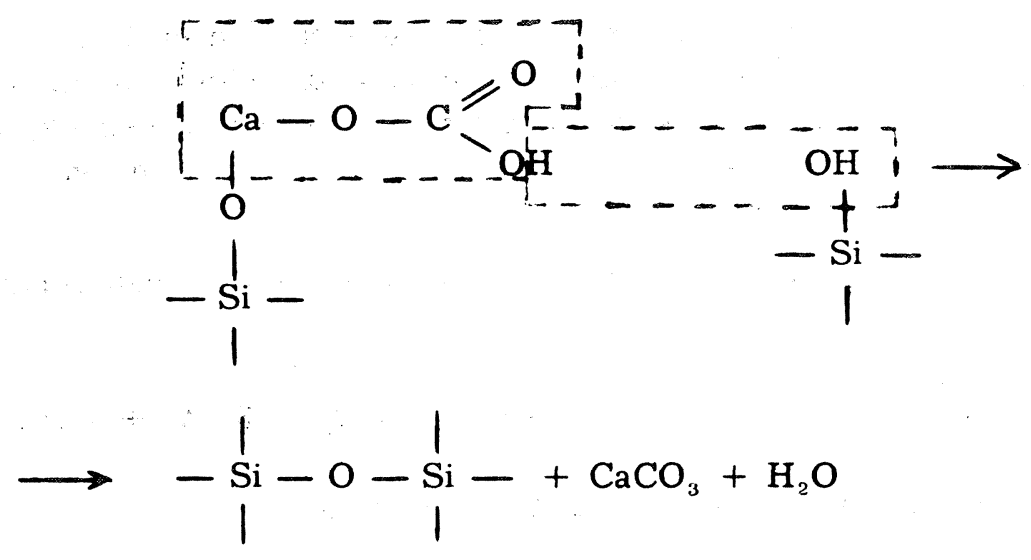

La unión entre el tetraedro de sílice y el Ca octaédrico se puede representar como sigue:

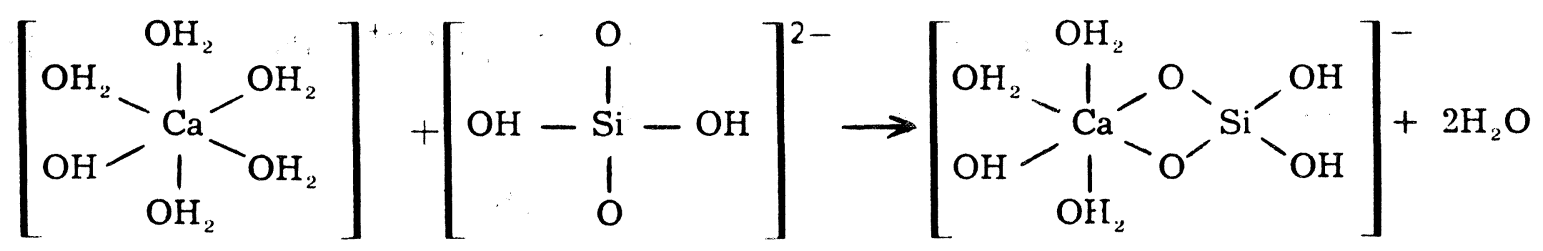


La existencia de silicoaluminatos se explica de la siguiente forma:

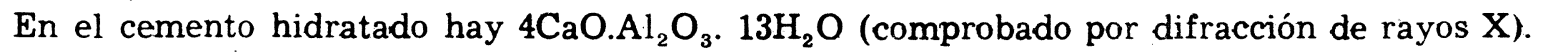

El equilibrio:

$$
4 \mathrm{CaO} \cdot \mathrm{Al}_{2} \mathrm{O}_{3} \cdot 13 \mathrm{H}_{2} \mathrm{O} \longleftrightarrow 2 \mathrm{AlO}_{4} \mathrm{H}_{5}+4 \mathrm{Ca}(\mathrm{OH})_{2}+4 \mathrm{H}_{2} \mathrm{O}
$$

o lo que es lo mismo:

$$
\left(\mathrm{AlO}_{4}\right)_{2} \cdot \mathrm{Ca}_{4} \mathrm{H}_{2} \cdot 12 \mathrm{H}_{2} \mathrm{O} \Longleftrightarrow 2 \mathrm{AlO}_{4} \mathrm{H}_{5}+4 \mathrm{Ca}(\mathrm{OH})_{2}+4 \mathrm{H}_{2} \mathrm{O}
$$

está desplazado hacia la izquierda en un medio fuertemente básico. La presencia de $\mathrm{CO}_{2}$ disminuye la basicidad del medio sucediendo simultáneamente las reacciones siguientes:

$$
\begin{array}{ll}
\mathrm{Ca}(\mathrm{OH})_{2}+\mathrm{CO}_{2} & \rightarrow \mathrm{CaCO}_{3}+\mathrm{H}_{2} \mathrm{O} \\
\mathrm{AlO}_{4} \mathrm{H}_{5} & \rightarrow \mathrm{Al}(\mathrm{OH})_{3}+\mathrm{H}_{2} \mathrm{O} \\
\mathrm{SiO}_{4}^{4-}+\mathrm{Al}^{3+} & \rightarrow\left(\mathrm{SiO}_{4}\right)_{3} \mathrm{Al}_{4}
\end{array}
$$

En la combinación [I] el aluminio está tetracoordinado. Al disminuir el pH, pasa a funcionar como catión hexacoordenado.

La presencia de silicato de aluminio se refleja en IR por las bandas de absorción en 1.280, 570 y $540 \mathrm{~cm}^{2}$ El $\mathrm{CaCO}_{3}$ de [II]. por las bandas específicas de calcita.

Por difracción de rayos $\mathrm{X}$ tan sólo se detecta en la muestra $\mathrm{CaCO}_{3}$, por lo que se puede deducir que el resto de las fases del cemento son amorfas o muy pobremente cristalinas.

Hemos encontrado efectos similares a los aquí citados, en hormigones industriales que estuvieron largo tiempo en contacto con medios agresivos

- Tratamiento con el método salicílico-metanol.

La figura 3 da los espectros IR de las muestras tratadas con el método salicilico-metanol (Takashima).

El espectro IR de la muestra $n .{ }^{\circ} 2$ presenta bandas de absorción en 1.150 y 1.085 (m. f.), 790 (d), $550(\mathrm{~m})$ y 460 (f) $\mathrm{cm}^{-1}$, que coinciden con mucha aproximación con las dadas por Moenke (7) para el $\mathrm{Al}_{2} \mathrm{O}_{3}$ en su variedad de "zafiro". En el espectro existen también además otras bandas características de Al-O (de Al tetracoordinado) y de yeso hemihidrato, principalmente en 602 y $660 \mathrm{~cm}^{-1}$.

La interpretación que se da, es la de descomposición del monosulfoaluminato, según la reacción siguiente:

$$
\mathrm{CaSO}_{4} \cdot \mathrm{Al}_{2} \mathrm{O}_{3} \cdot 3 \mathrm{CaO} \cdot 12 \mathrm{H}_{2} \mathrm{O} \rightarrow \mathrm{CaSO}_{4} \cdot \frac{1}{2} \mathrm{H}_{2} \mathrm{O}+\mathrm{Al}_{2} \mathrm{O}_{3}+3 \mathrm{CaO}+11,5 \mathrm{H}_{2} \mathrm{O}
$$

distinguiéndose en cada muestra el tipo de $\mathrm{Al}_{2} \mathrm{O}_{3}$ obtenido.

En el espectro de la muestra n. 3 tratada con el método de Takashima (figura 3 ; curva n. ${ }^{\circ}$ 3) aparecen bandas de absorción que indican la existencia de una notable cantidad de $\mathrm{CaCO}_{3}$ (calcita) y una variedad de $\mathrm{Al}_{2} \mathrm{O}_{3}$ de transición (bandas en 1.150, 1.050 y $310 \mathrm{~cm}^{-1}$ ).

Según Henning (8) la destrucción del monosulfoaluminato cálcico puede formularse como sigue:

$$
\mathrm{CaSO}_{4} \cdot \mathrm{Al}_{2} \mathrm{O}_{3} \cdot 3 \mathrm{CaO} \cdot 12 \mathrm{H}_{2} \mathrm{O} \rightarrow \mathrm{C}_{3} \mathrm{AH}_{6}+\mathrm{CaSO}_{4} \cdot 2 \mathrm{H}_{2} \mathrm{O}+4 \mathrm{H}_{2} \mathrm{O}
$$

Nosotros hemos observado que el yeso se deshidrata según:

$$
\mathrm{CaSO}_{4} \cdot 2 \mathrm{H}_{2} \mathrm{O} \rightarrow \mathrm{CaSO}_{4} \cdot \frac{1}{2} \mathrm{H}_{2} \mathrm{O}+1,5 \mathrm{H}_{2} \mathrm{O}
$$


y que no existe $\mathrm{C}_{3} \mathrm{AH}_{6}$ en las muestras estudiadas, lo que se interpreta por la acción del $\mathrm{CO}_{2}$, según las secuencias siguientes:

$$
\begin{array}{ll}
\mathrm{C}_{3} \mathrm{AH}_{6}+\mathrm{CO}_{2} & \rightarrow \mathrm{C}_{3} \mathrm{AH}_{4} \cdot \mathrm{CO}_{2}+2 \mathrm{H}_{2} \mathrm{O} \\
\mathrm{C}_{3} \mathrm{AH}_{4}\left(\mathrm{CO}_{2}\right)+\mathrm{CO}_{2} & \rightarrow \mathrm{C}_{3} \mathrm{AH}_{2}\left(\mathrm{CO}_{2}\right)_{2}+2 \mathrm{H}_{2} \mathrm{O} \\
\mathrm{C}_{3} \mathrm{AH}_{2}\left(\mathrm{CO}_{2}\right)_{2}+\mathrm{CO}_{2} & \rightarrow \mathrm{C}_{3} \mathrm{AH}\left(\mathrm{CO}_{2}\right)_{3}+2 \mathrm{H}_{2} \mathrm{O} \\
\mathrm{C}_{3} \mathrm{~A}\left(\mathrm{CO}_{2}\right)_{3} & =3 \mathrm{CaCO}_{3}+\mathrm{Al}_{2} \mathrm{O}_{3}
\end{array}
$$

Los compuestos resultantes de las reacciones [VIII], [IX] y [X] han sido estudiadas por los autores del presente trabajo en colaboración con F. Triviño (9), y han sido denominados por nosotros " $\mathrm{CO}_{2}$-hidrogranates".

Por otra parte no aparecen en el espectro IR n." 3 las bandas débiles de $\mathrm{AlO}_{4}$, que sí estaban en el $n .{ }^{\circ} 2$, lo que se interpreta de la misma manera que se hacía en las muestras no tratadas: existencia de silicoaluminatos cálcicos hidratados en la muestra n. 2 y ausencia de éstos en la n. 3 . En el espectro de aquélla se observan bandas $\left(570,540 \mathrm{~cm}^{-1}\right)$ características de $\mathrm{Al}$ en coordinación octaédrica.

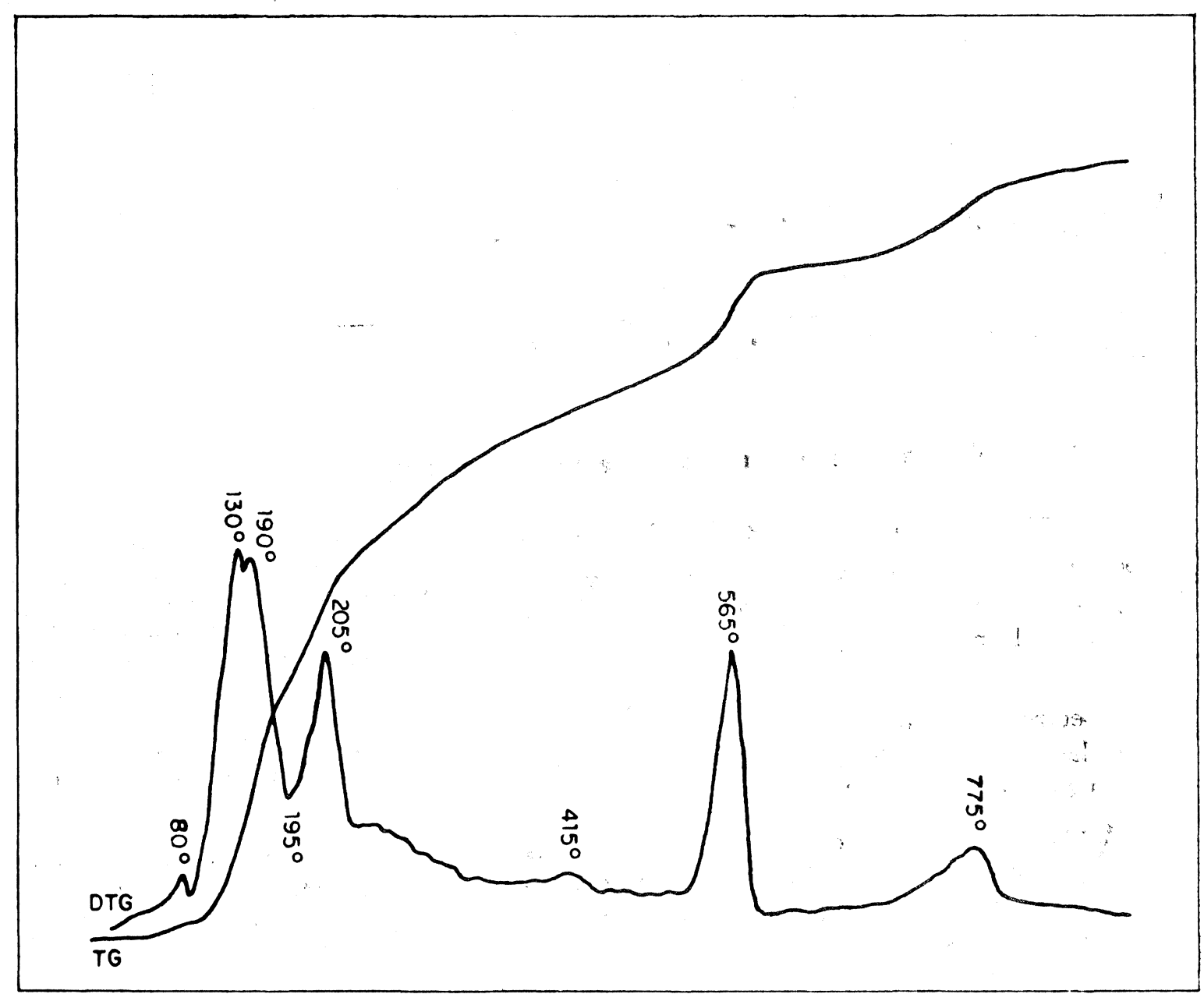

Fig. 7.-A.T. del cemento hidratado aceleradamente. Ambiente de $\mathrm{CO}_{2}$.

El análisis térmico de la muestra n." 3 (fig. 8) confirma lo interpretado por espectroscopía IR. 


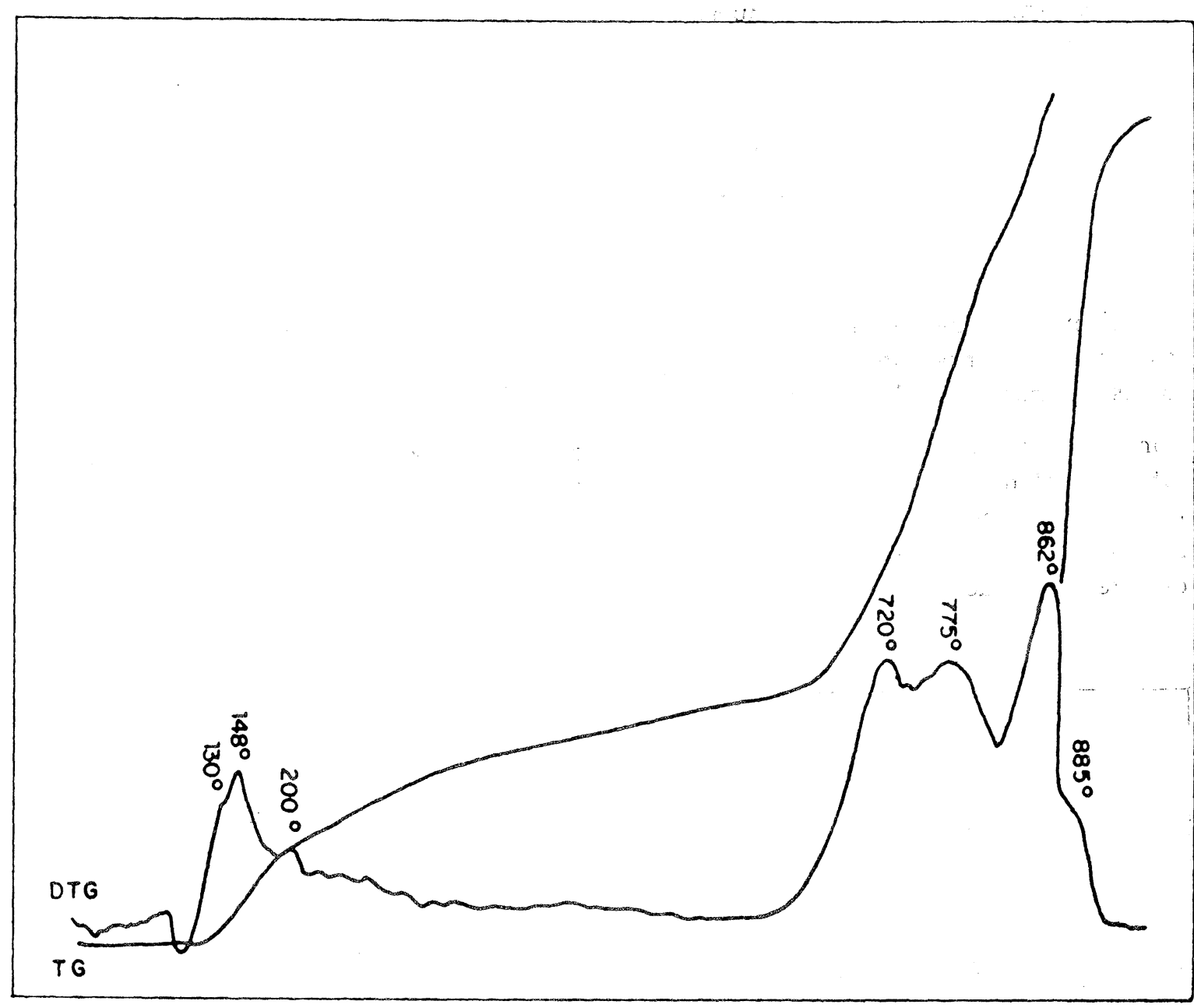
Fig. 8.- - A.T. del cemento hidratado aceleradamente. Ambiente de $\mathrm{CO}_{2}$ y posteriormente mantenido en ambiente
saturado de $\mathrm{CO}_{2}$.

\subsection{Cemento hidratado aceleradamente en ambiente de $\mathrm{CO}_{2}$}

El espectro n." 1' (figura 2) corresponde a la muestra del cemento hidratado normalmente. El espectro $n .^{\circ} 2$, que corresponde a la muestra hidratada aceleradamente en ambiente de $\mathrm{CO}_{2}$, presenta un gran parecido con el estudiado en el 3.1. La diferencia más notable estriba en la mayor intensidad de las bandas de carboaluminatos en el espectro de la muestra 2'.

En el espectro n. 3 ' hay (respecto al n. ${ }^{\circ}$ ) pequeñas, pero importantes diferencias: no aparecen las bandas débiles asignadas a $\mathrm{AlO}_{6}$ y $\mathrm{Si}-\mathrm{O}-\mathrm{Al}$. La banda característica de la tobermorita está en $1.025 \mathrm{~cm}^{-1}$.

La interpretación de estas diferencias podría ser la siguiente: En la muestra 3 (ambiente $\mathrm{N}_{2}$ ) hay sulfoaluminatos cálcicos y aluminato tetracálcico hidratado y su carbonatación transcurre según el proceso [VI]-[XI], mientras que en la 3' (ambiente de $\mathrm{CO}_{2}$ ) lo hace según el proceso siguiente:

1." Hay una sustitución de 2 de $\mathrm{H}_{2} \mathrm{O}$ por $1 \mathrm{CO}_{2}$ :

$$
3 \mathrm{CaO} \cdot \mathrm{Al}_{2} \mathrm{O}_{3} \cdot \mathrm{CaCO}_{3} \cdot 12 \mathrm{H}_{2} \mathrm{O}+\mathrm{CO}_{2} \rightarrow 3 \mathrm{CaO} \cdot \mathrm{Al}_{2} \mathrm{O}_{3} \cdot \mathrm{CaCO}_{3} \cdot \mathrm{CO}_{2} \cdot 10 \mathrm{H}_{2} \mathrm{O}+2 \mathrm{H}_{2} \mathrm{O}
$$

o lo que supone, al reaccionar estequiométricamente el $\mathrm{CO}_{2}$ con $\mathrm{CaO}$ del aluminato tricálcico (que por otra parte, permanece en la relación $3 \mathrm{CaO} / 1 \mathrm{Al}_{2} \mathrm{O}_{3}$ ):

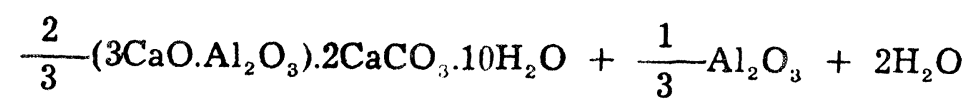


2." Se sustituyen $\frac{2}{3} \mathrm{CO}_{2}$ por $\frac{4}{3}$ de $\mathrm{H}_{2} \mathrm{O}$, por lo que, una vez supuesta la reacción del $\mathrm{CO}_{2}$ con $\mathrm{CaO}$ y la liberación de $\mathrm{Al}_{2} \mathrm{O}_{3}$, queda:

$$
\frac{4}{9}\left(3 \mathrm{CaO} \cdot \mathrm{Al}_{2} \mathrm{O}_{3}\right) \cdot \frac{8}{3} \mathrm{CaCO}_{3} \cdot \frac{26}{3} \mathrm{H}_{2} \mathrm{O}+\frac{5}{9} \mathrm{Al}_{2} \mathrm{O}_{3}+\frac{4}{3} \mathrm{H}_{2} \mathrm{O}
$$

3. $\quad$ La siguiente reacción quedaría:

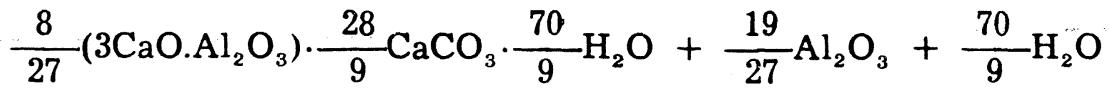

En definitiva, los coeficientes dan sendas sucesiones, de límites:

$$
\begin{aligned}
& \text { Para el del } 3 \mathrm{CaO} \cdot \mathrm{Al}_{2} \mathrm{O}_{3}=0 \\
& \text { Para el del } \mathrm{CaCO}_{3} \quad=4 \\
& \text { Para el del } \mathrm{H}_{2} \mathrm{O} \quad=6 \\
& \text { Para el del } \mathrm{Al}_{2} \mathrm{O}_{3} \quad=1
\end{aligned}
$$

Es decir, el resultado final será:

$$
\left(3 \mathrm{CaO} \cdot \mathrm{Al}_{2} \mathrm{O}_{3}\right) \cdot \mathrm{CaCO}_{3} \cdot 12 \mathrm{H}_{2} \mathrm{O}+3 \mathrm{CO}_{2} \rightarrow \mathrm{Al}_{2} \mathrm{O}_{3}+4 \mathrm{CaCO}_{3}+6 \mathrm{H}_{2} \mathrm{O}+6 \mathrm{H}_{2} \mathrm{O}
$$

De alguna forma existen 6 moles de agua de distinta naturaleza a los otros restantes 6 moles.

- Tratamiento con el método salicílico-metanol.

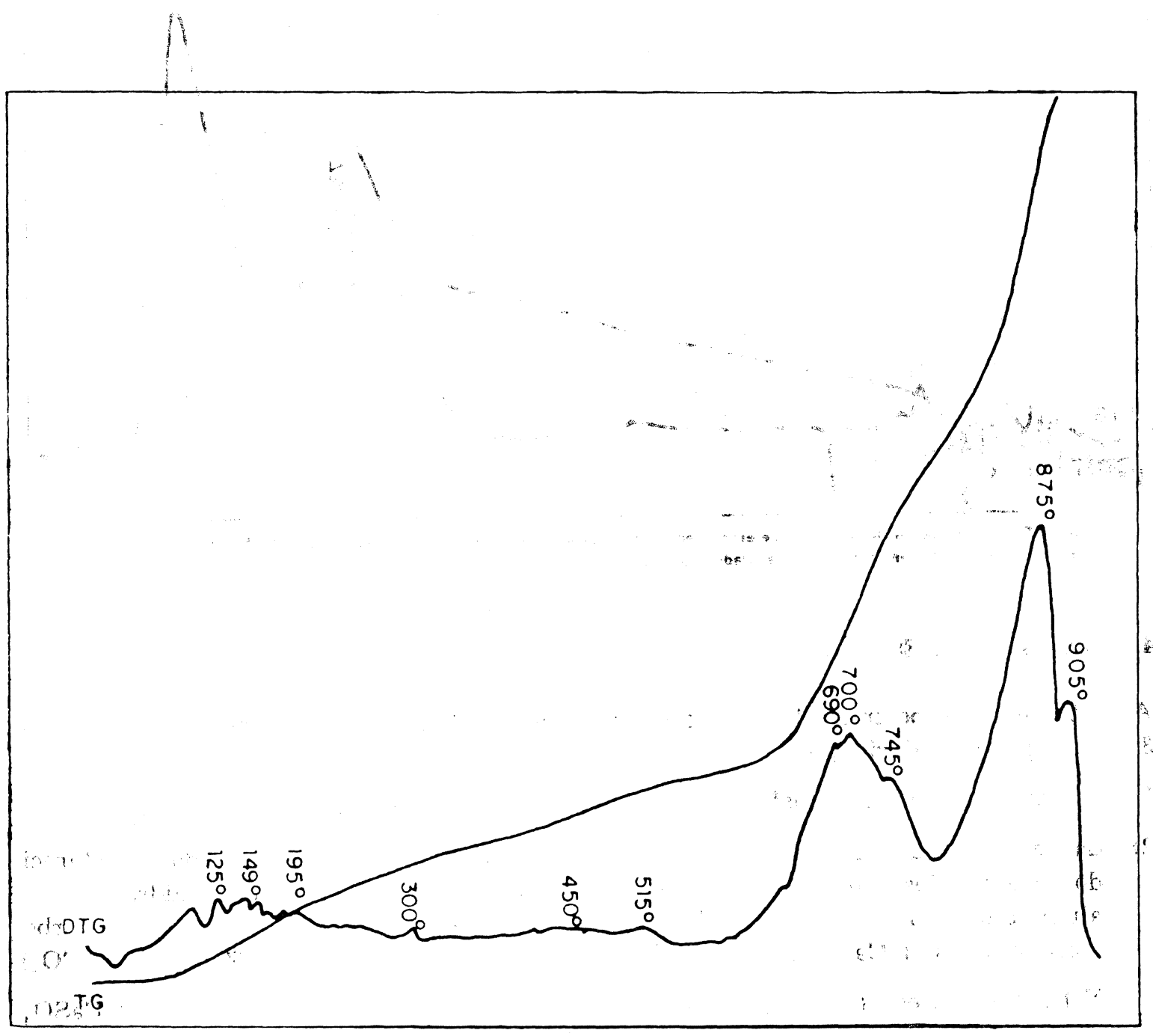

Fig. 9.-A.T. del cemento hidratado aceleradamente y tratado con el método atakashiman. Ambiente de $N_{2} y$ posteriormente mantenido en ambiente saturado de $\mathrm{CO}_{2}$. 
En la figura 4 se dan los espectros IR de las muestras inicialmente carbonatadas y ti atadas con el método de Takashima. La muestra 2' presenta un espectro similar a la 2, ya estudiada.

El espectro IR correspondiente a la muestra 3' se diferencia de la muestra 3 tan sólo por una mayor absorción de las bandas carácterísticas de $\mathrm{CaSO}_{4} \cdot \frac{1}{2} \mathrm{H}_{2} \mathrm{O}$ producido según la reacción [VII] de 3.1. La figura 10 presenta el correspondiente termograma.

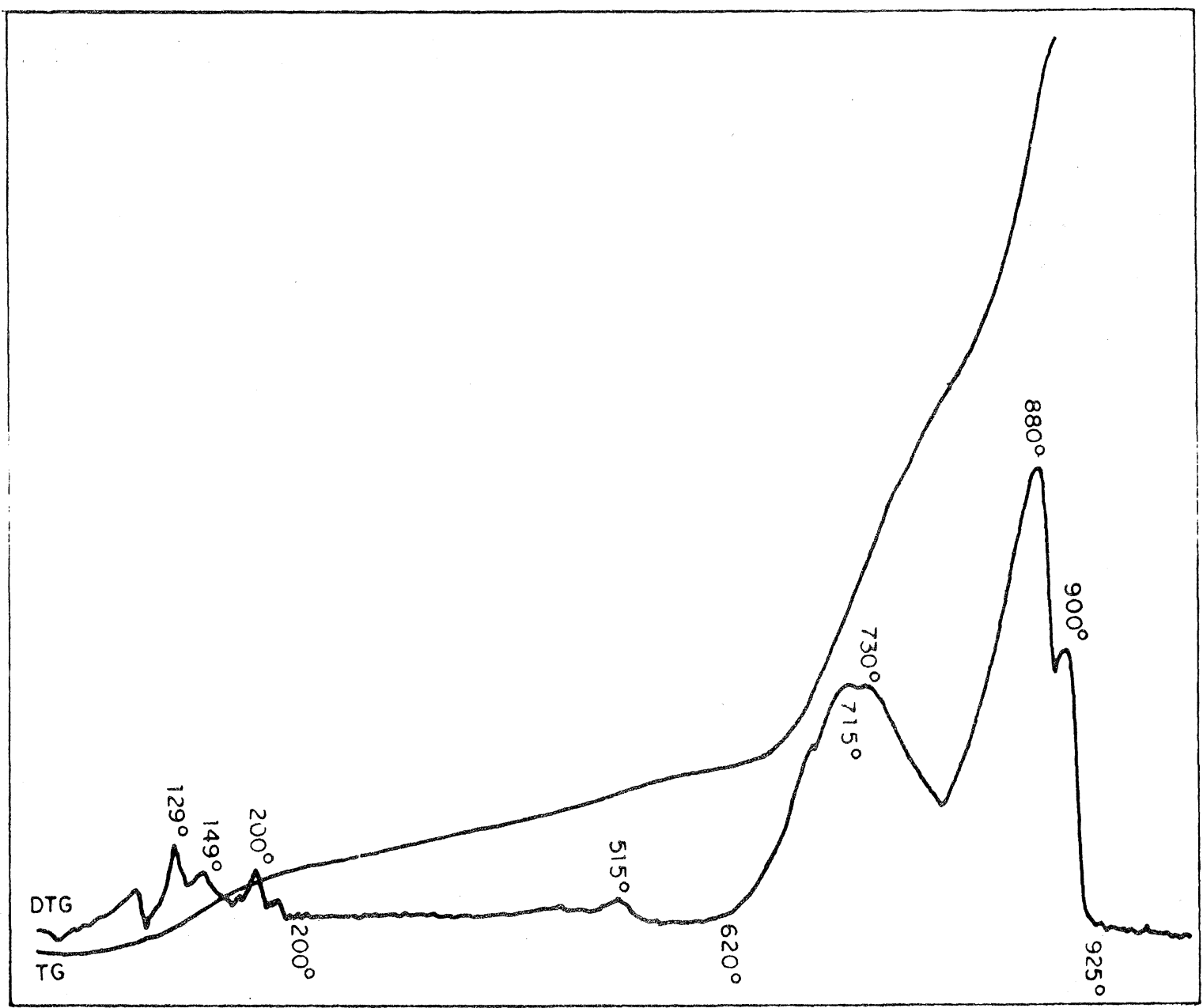

Fig. 10.-A.Y. del cemento hidratado aceleradamente $y$ tratado con el método "Takashiman. Ambiente de $\mathrm{CO}_{2} \mathrm{Y}$ posferiormente mantenido en ambiente saturado de $\mathrm{CO}_{2}$.

\section{CONCLUSIONES}

A partir de los espectros IR de los cementos portland hidratados aceleradamente durante 3 días se puede deducir que:

1) La ettringita se ha transformado en monosulfoaluminato tetracálcico hidratado.

2) La acción de un ambiente saturado de $\mathrm{CO}_{2}$ sobre el cemento hidratado y endurecido hace que los sulfoaluminatos se transformen en sulfato cálcico dihidrato $\mathbf{y}$ en aluminato tricálcico cúbico $\left(3 \mathrm{CaO}_{2} \mathrm{O} \cdot \mathrm{Al}_{2} \mathrm{O}_{3} \cdot 6 \mathrm{H}_{2} \mathrm{O}\right)$. Este se convierte después en "carbo-

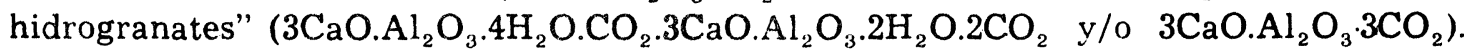
$\mathrm{Y}$ finalmente, éstos dan lugar a $\mathrm{Al}_{2} \mathrm{O}_{3}$ ("zafiro"), $\mathrm{CaCO}_{3}$ (calcita) y $\mathrm{H}_{2} \mathrm{O} . \mathrm{El} \mathrm{CaSO}$. $.2 \mathrm{H}_{2} \mathrm{O}$ pasa entretanto a $\mathrm{CaSO}_{4} \cdot \frac{1}{2} \mathrm{H}_{2} \mathrm{O}$. 
3) El $\mathrm{CO}_{2}$ inicialmente existente en el agua de amasado y en el cemento anhidro ("meteorizado") impide la formación de silicoaluminatos cálcicos. Se ha demostrado que este comportamiento se debe a la formación previa de carboaluminatos y carbosilicatos cálcicos.

4) $\mathrm{El} \mathrm{CO}_{2}$ ejerce acción polimerizante sobre las fases hidratadas del cemento. La acción sobre la tobermorita y los aluminatos cálcicos hidratados es gradual.

\section{B I B L I O G R A F I A}

(1) Vazquez, T. y Gaspar, D.: Materiales de Construcción, n. ${ }^{\circ}$ 161, enero-febrero-marzo (1976).

(2) Vazquez, T. y Gaspar, D.: Materiales de Construcción, n. ${ }^{\circ}$ 162, abril-mayo-junio (1976).

(3) VAzQuez, T.: Materiales de Construcción, n. 163 , julio-agosto-septiembre (1976).

(4) Vazquez, T., Calleja, J. y Triviño, F.: Materiales de Construcción, n. ${ }^{\circ}$ 164, junio-agosto-septiembre (1976).

(5) SAgrera, J. L.: Materiales de Construcción, n. 145, enero-febrero-marzo (1972).

(6) TAkashima, S y. Kato, M. P.: 33 Review of the Fifteenth General Meeting (1961).

(7) Moenke, H.: Mineralspektren. Akademie Verlag. Berlín (1962).

(8) Henning, O. y Danowski, W.: Silikattechnik, 325, 17 (1966).

(9) Vazquez, T. Triviño, F. y Ruiz de Gauna, A.: Materiales de Construcción, núms. 157,158 y 159 (1975). 